
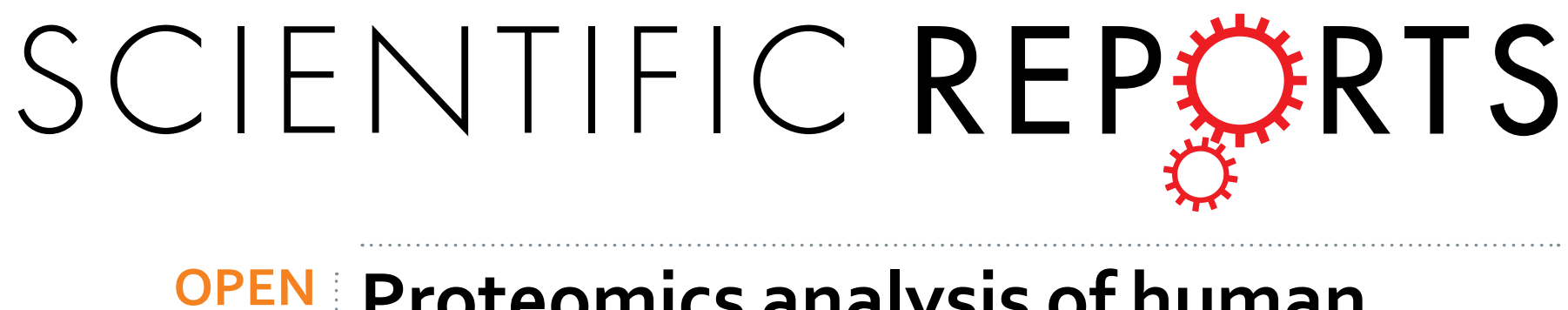

\title{
Proteomics analysis of human tears from aqueous-deficient and evaporative dry eye patients
}

Received: 21 March 2016

Accepted: 20 June 2016

Published: 20 July 2016

\section{Natarajan Perumal, Sebastian Funke, Norbert Pfeiffer \& Franz H. Grus}

Despite the high global prevalence of dry eye syndrome (DES), the fundamental processes underlying this pathology remain largely unexplored. Therefore, this study endeavoured to investigate indepth the tear proteome of DES patients employing the mass spectrometry (MS)-based proteomic strategies. Eighty patients were recruited and subdivided into three major DES subgroups, which are the aqueous-deficient (DRYaq), evaporative (DRYlip) and a combination of the two (DRYaqlip), as well as healthy subjects (CTRL). Discovery proteomics strategy was employed to identify large number of significantly differentially expressed tear proteins in DRYlip vs. CTRL, DRYaq vs. CTRL and DRYaqlip vs. CTRL with 22, 58 and 67 proteins, respectively. Biological functional analysis demonstrated for the first time that various metabolic processes were highly expressed in DRYaq and DRYaqlip, which might modulate various other known processes, especially the inflammatory and immune processes. Targeted proteomics strategy verified that 13 major proteins were differentially expressed in specific DES subgroups, comprising of PRR4, ZG16B, SCGB2A1, DMBT1, PROL1, LACRT, ALDH3A1, ENO1, TF, S100A8, S100A9, PEBP1 and ORM1. In conclusion, this study had explored in-depth the pathology of DES by unravelling various new fundamental processes and the major proteins responsible for the maintenance of tear film stability.

It is well- recognized that the dry eye syndrome (DES) is a common yet deplorable pathology of the ocular surface and tears that arises from a plethora of factors, which could result in decreased visual acuity, ocular discomfort and tear film instability with potential damage risk to the cornea ${ }^{1}$. The study of DES has become a subject of much interest due to the high rate of occurrence in different ethnic groups, gender (preponderance in women), age ( $>50$ years old) and other ocular surface-affecting systemic diseases (especially Sjögren's syndrome, diabetes mellitus, pterygium and allergy ${ }^{2-7}$. In recent years, assessment studies in several Western and Asian countries reported a high global prevalence of this pathology, ranging from $2 \%$ to over $50 \%$ of the world population ${ }^{8-17}$. Apart from afflicting one out of every two elderly in some populations, this condition also poses a substantial economic burden on the health system ${ }^{18,19}$. There are various underlying aetiologies for DES and the major causes of DES are a consequence of aqueous tear-deficiency (DRYaq) or due to changes in the lipid phase (DRYlip) of the tear film ${ }^{1}$.

To date, many studies have identified the differentially expressed proteins in tears of DES patients. Grus et al. had profiled tear proteins from patients with DES and they found a significant increase in S100-A8 protein (S100A8) and decrement of proline-rich protein 4 (PRR4), lysozyme C (LYZ), proline-rich protein 3 and $\alpha-1$-antitrypsin ${ }^{20}$. The investigation of the tear proteome of patients with DRYaq, DRYlip and a combination of the two (DRYaqlip) compared to healthy controls (CTRL) by Boehm et al. revealed that specific alterations of the tear proteome reflect the different clinical phenotypes of $\mathrm{DES}^{21}$. They also demonstrated that PRR4 expression level was decreased and, 3 other proteins, including S100A8, increased significantly in both DRYaq and DRYaqlip groups. However, the tear proteome of DRYlip patients strongly deviated from the DRYaq or DRYaqlip groups and demonstrated only slight alterations. Meanwhile, Soria et al. showed that 6 proteins were found to be increased and 9 proteins to be decreased in abundance in both DRYaq and DRYlip ${ }^{22}$. Zhou et al. employed the isobaric Tags for Relative and Absolute Quantitation (iTRAQ) quantitative proteomics strategy to identify potential tear biomarkers for DES and they found an increase of 6 proteins and a decrease of 4 proteins ${ }^{23}$. Similarly, utilizing the iTRAQ tool, Srinivasan et al. investigated the differentially expressed tear proteins in patients with mildly symptomatic aqueous deficiency (MDE), symptomatic aqueous deficiency (MSDE) and a combination group $(\mathrm{MXDE})^{24}$. In addition to the decreased abundance of commonly reported proteins, such as LYZ, lipocalin-1 
(LCN1) and prolactin-inducible protein (PIP) across all DES sub-groups, their finding demonstrated a number of proteins that were significantly differentially expressed in subgroups of DES. Other DES studies have investigated Sjögren's syndrome (SS) dry eye, which is a systemic autoimmune disease characterized by compromised lacrimal and salivary gland functions that causes severe dry eye and dry mouth ${ }^{6,25}$.

The aforementioned DES studies only reported limited number of differentially expressed proteins for the pathology, largely due to the limitations in the methodology employed. These studies had employed various mass spectrometry (MS)-based proteomic strategies, which have become a progressively powerful technology for the identification and quantifications of tear proteins in different ocular pathologies ${ }^{5,20,21,23,26-35}$. Recently, protein quantification with isotopic labels (e.g. iTRAQ) has been commonly utilized for tear protein studies due to their quantitative accuracy, coverage and robustness ${ }^{23,24}$. However, despite their usefulness, they inherently require extra preparation steps that consequently increase the complexity and often prohibit their use for biological questions that require the detection of subtle yet vital proteome changes, mostly the low abundant tear proteins. On the contrary, label-free quantification (LFQ) is the simplest and most economical strategy that can be utilized for in-depth analysis of the differentially expressed proteins in large sample size with high precision ${ }^{36}$. Additionally, the development and improvement of the existing algorithms and software tools, especially, MaxQuant software suite, have provided an effective solution for LFQ analysis, one that achieves state-of-the-art quantification accuracy and coverage ${ }^{37,38}$.

Therefore, in-depth investigation of the differentially expressed proteins in tear proteome of DES patients will potentially contribute to better understanding of the fundamental processes of this pathology. Hence, the main objective of the present study was to identify the differentially expressed proteins in the major DES subgroups, which are responsible for the maintenance of tear film stability. The tear proteome profiles of DES subgroups were analysed employing the bottom-up label-free MS-based proteomics strategies. The ultimate outcome of this study is envisaged to unravel the intricate regulation profiles of specific proteins responsible for the maintenance of tear film stability and pin-point specific alteration(s) in the different DES subgroups.

\section{Results}

Identification of the differentially expressed tear proteins from DES patients. The representative tear protein profiles of DES subgroups and CTRL resolved in 1DE gel are illustrated in Fig. 1a. Notable differences were observed at band three, four, seven and ten, which represent serum albumin (ALB), Ig alpha-1 chain $\mathrm{C}$ (IGHA1), PRR4 and mammaglobin-B (SCGB2A1), respectively. A total of 200 proteins were detected by the discovery approach as presented in Supplementary data 1A. In order to reveal the differentially expressed proteins in the DES subgroups compared to CTRL, LFQ values of the identified proteins extracted from MaxQuant analysis were used for statistical analysis utilizing the Perseus software. Basically, there is good technical reproducibility of the entire experimental workflow, which reveals Pearson correlation of $0.96 \pm 0.01$ for DRYaq and $0.97 \pm 0.01$ for CTRL, DRYlip and DRYaqlip as shown in Fig. 1b. On average, correlation between DRYlip vs. CTRL was $0.96 \pm 0.01$, and slightly lower correlations were observed between the DRYaq vs. CTRL $(0.89 \pm 0.01)$ and DRYaqlip vs. CTRL $(0.87 \pm 0.01)$ groups. Hence, this demonstrated high similarities between the proteome of CTRL and DRYlip, as well as between DRYaq and DRYaqlip. In addition, this analysis indicates that reproducible data were generated from the pooled tear samples, which enabled further statistical analysis. A heat map with unsupervised hierarchical clustering of the data was generated and resulted in two major clusters, which are cluster 1 comprising CTRL and DRYlip, and cluster 2 comprising DRYaq and DRYaqlip, as shown in Fig. 1c. In order to identify a subset of proteins that significantly differentiate the DES subgroups compared to CTRL, a two samples t-test $(\mathrm{P}<0.01)$ was performed. The summary of the significantly differentially expressed proteins in the different DES subgroups compared to CTRL is as tabulated in Supplementary data 1B. The total number of proteins that were significantly differentially expressed in the DRYlip vs. CTRL was 22 proteins, 58 proteins in the DRYaq vs. CTRL and 67 proteins in the DRYaqlip vs. CTRL. Next, intensity-based absolute quantification (iBAQ) values of the identified proteins extracted from MaxQuant analysis were used to elucidate the abundance of the identified proteins between DES subgroups and CTRL. Figure 1d shows that only top 20 abundant proteins from the total of 200 proteins identified make up almost $95.52 \pm 0.32 \%, 96.36 \pm 0.65 \%, 91.0 \pm 0.13 \%$ and $92.55 \pm 0.56 \%$ of the total abundance in CTRL, DRYlip, DRYaq and DRYaqlip, respectively (complete data in Supplementary data 1A). Moreover, LYZ, LCN1, lactotransferrin (LTF) and PIP make up as much as $60 \%$ of the total abundance of the CTRL tears. Generally, the aforementioned iBAQ analysis revealed that most of the significantly differentially expressed proteins were classified as low abundant proteins (lower than $0.5 \%$ ) in this study, as shown in Supplementary data $1 \mathrm{~B}$.

Functional classification and protein interaction network analysis of the significantly altered proteins. To determine which biological processes were most affected in specific DES subgroups, the over-represented gene ontology biological process (GOBP) terms associated with the differentially expressed proteins were analysed employing the Database for Annotation, Visualization and Integrated Discovery (DAVID) tool. Several major biological process categories were observed to be decreased in DRYlip vs. CTRL, mainly inflammatory response (4\%), however they were identified with lower percentage, as shown in Fig. 2a. On the contrary, 4 major biological processes categories were observed to be decreased in DRYaq vs. CTRL, especially immune responses (60\%), as shown in Fig. 2b. Consequently, 18 major biological processes were found to be increased in this group and large percentage of them are involved in inflammatory response (51\%), catabolic process $(49 \%)$, cell death (38\%), response to wounding (35\%), defence response (32\%), metabolic process $(32 \%)$ and apoptosis (19\%). Six major biological processes were found to be decreased in DRYaqlip vs. CTRL, especially immune response (60\%) and defence response (32\%), as shown in Fig. 2c. Meanwhile, as many as 30 biological processes were found to be increased in this group and most highly ranked were involved in cell death (83\%), metabolic process (66\%), inflammatory response (49\%), catabolic process (46\%) and apoptosis (41\%). 
(a)

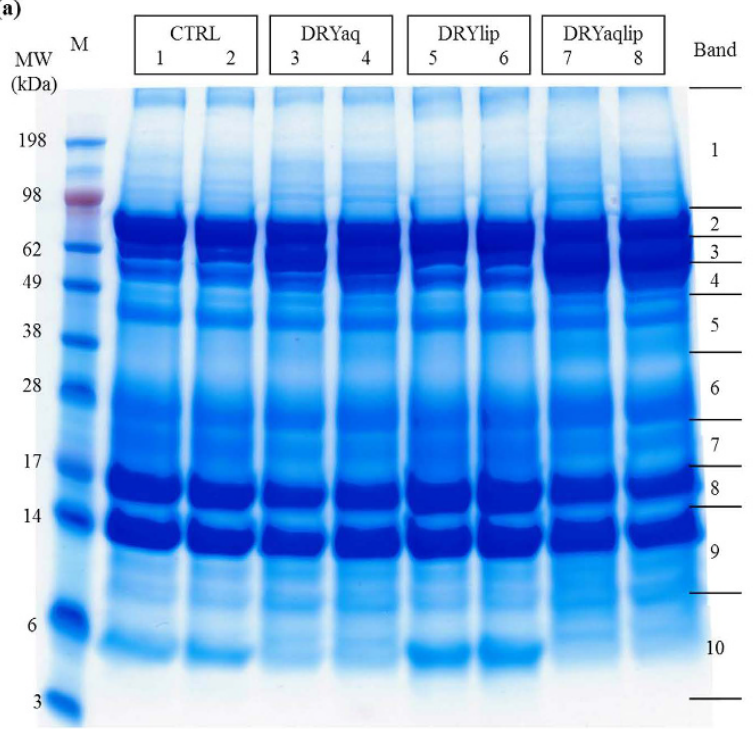

(b)

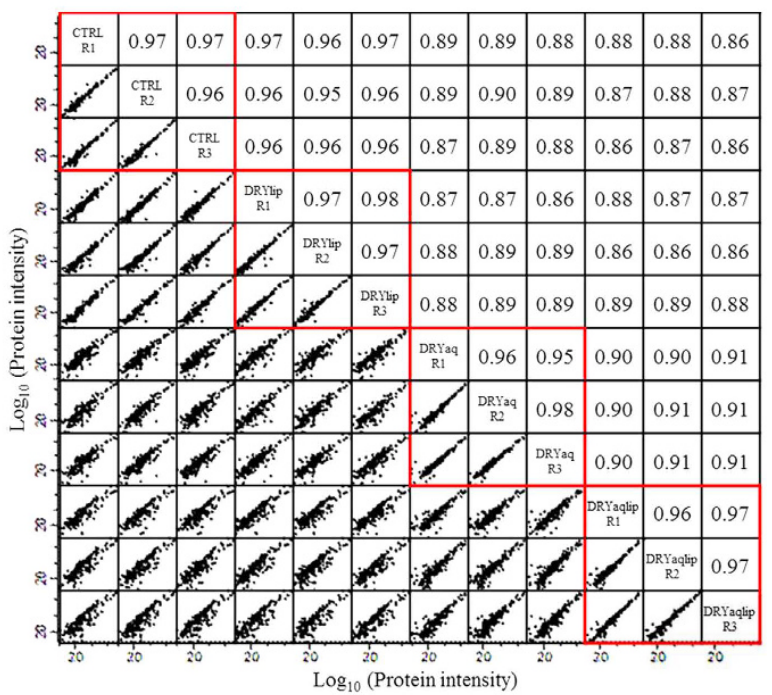

(c)
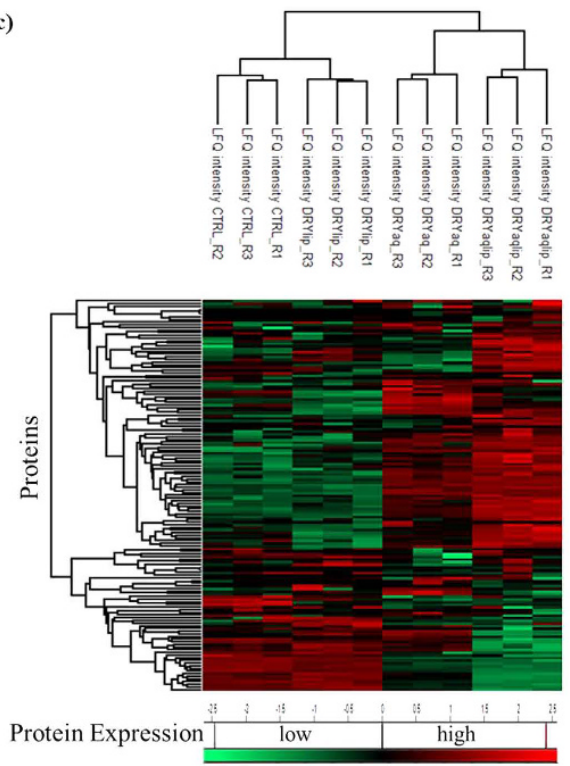

(d)

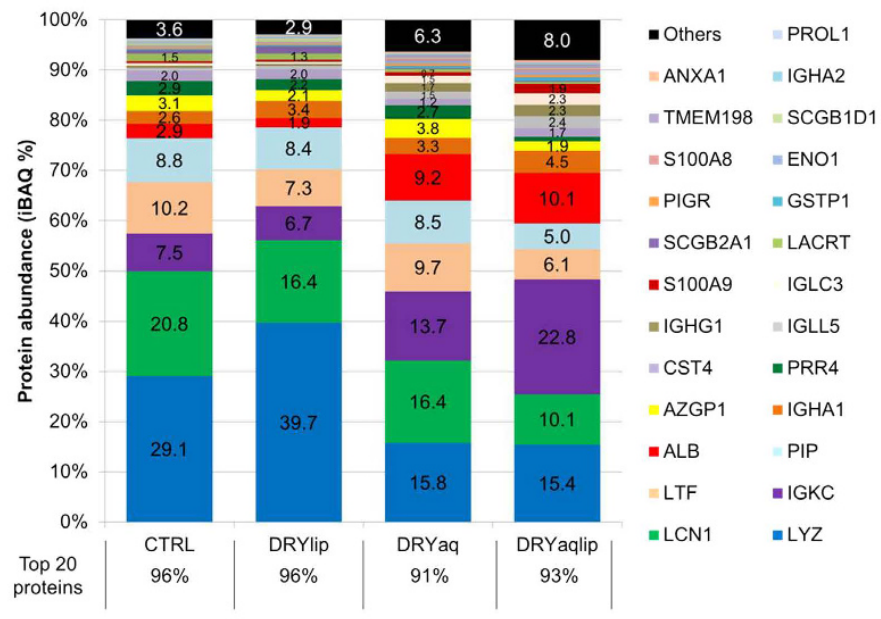

Figure 1. Discovery proteomics analysis via1DE \& LC-ESI-MS/MS strategy reveals the characteristics of the tear proteome of DES patients. (a) Representative tear protein profiles of the DES subgroups and CTRL resolved in 1DE gel after colloidal blue staining. (b) The degree of variances in the proteome between the DES subgroups and CTRL investigated by Pearson correlation analysis. (c) The heat map shows hierarchical clustering analysis of the 200 tear proteins that separates the designated groups into two main clusters. (d) The bar chart shows the degree of protein abundance in DES subgroups and CTRL.

The complete list of the over-represented GOBP terms of the differentially expressed proteins in DES subgroups compared to CTRL can be found in Supplementary data 1C. The characteristic of the differentially expressed proteins were further analysed, the over-represented gene ontology cellular component (GOCC) terms, molecule types and protein-protein interaction (PPI) networks were generated using Ingenuity Pathways Analysis. The over-represented GOCC terms of the significantly decreased proteins in DRYlip vs. CTRL were 5 extracellular space proteins and 9 cytoplasm proteins as well as increased in other 5 extracellular space proteins as shown in Fig. 3a. Meanwhile, the most significantly decreased proteins in DRYaq vs. CTRL were 8 extracellular space proteins and 12 cytoplasm proteins as well as drastic increased in other 12 extracellular space proteins and as many as 19 cytoplasm proteins as shown in Fig. 3b. Almost similar profiles were observed in DRYaqlip vs. CTRL, decreased of 13 extracellular space proteins and 12 cytoplasm proteins as well as increased in other 10 extracellular space proteins and 24 cytoplasm proteins were identified as shown in Fig. 3c. The molecular characteristics analysis of the differentially expressed proteins showed that many of these proteins are enzyme (23 proteins), transporter (12 proteins), kinase ( 2 proteins) peptidase ( 3 proteins), growth factor ( 1 protein), transmembrane receptor ( 1 protein), phosphatase (1 protein) and other (36 proteins), as shown in Supplementary data 1B. Generally, only numerous proteins of enzyme, transporter and peptidase were observed to be differentially expressed in DRYlip 

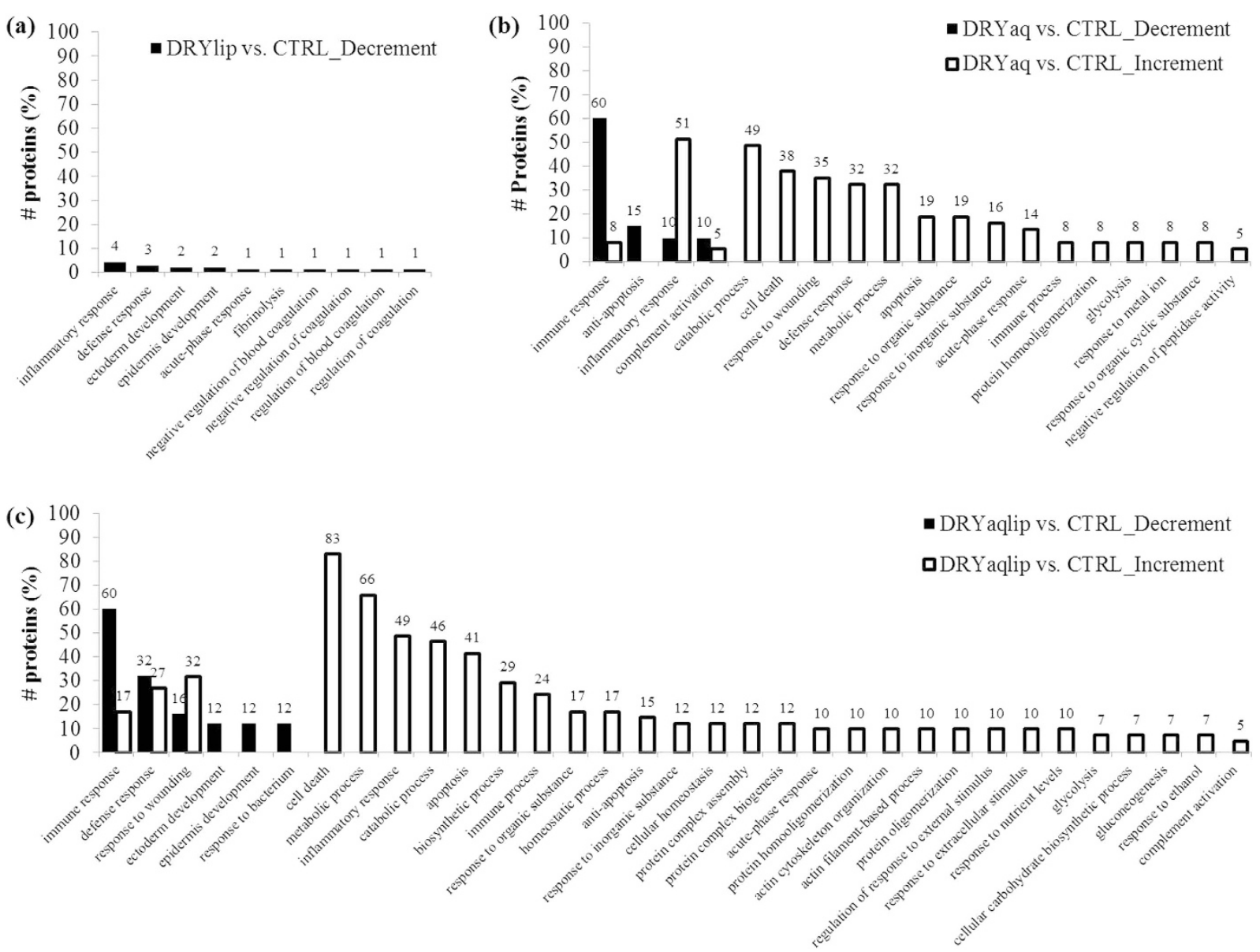

Figure 2. The bar charts shows the over-represented GOBP terms associated with the differentially expressed proteins analysed employing the DAVID tool in (a) DRYlip vs. CTRL, (b) DRYaq vs. CTRL and (c) DRYaqlip vs. CTRL.

(a) DRYlip vs. CTRL

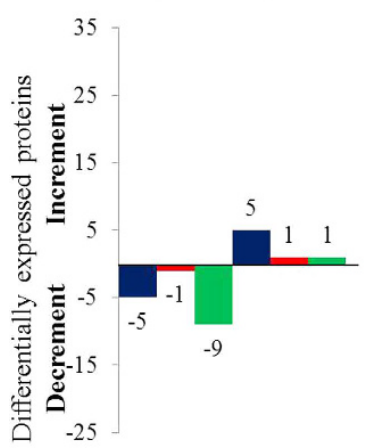

(b) DRYaq vs. CTRL

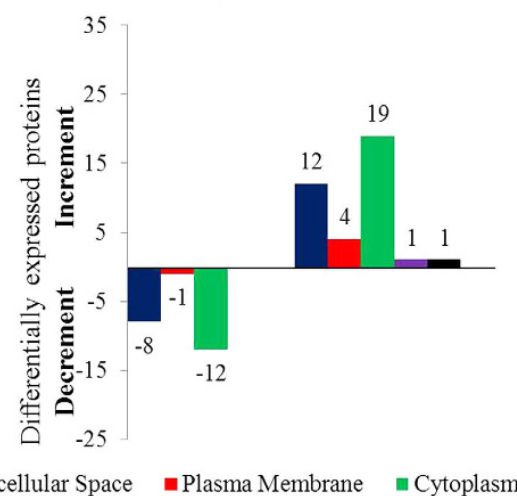

(c) DRYaqlip vs. CTRL

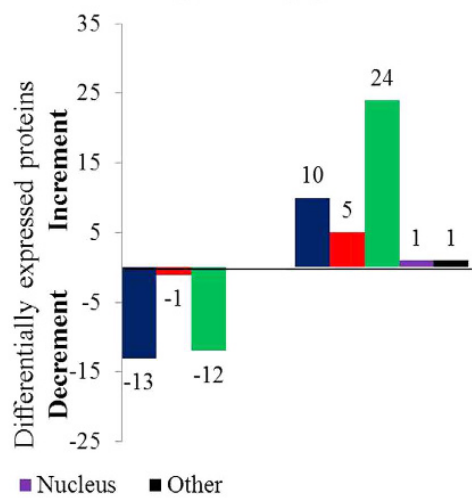

Figure 3. The bar charts shows the over-represented GOCC terms associated with the differentially expressed proteins analysed employing the Ingenuity Pathways Analysis software in (a) DRYlip vs. CTRL, (b) DRYaq $v s$. CTRL and (c) DRYaqlip vs. CTRL.

vs. CTRL, as shown in Fig. 4a. On the contrary, large preponderances of enzyme (12 proteins) and transporter (8 proteins) proteins were observed to be increased in DRYaq $v s$. CTRL, as shown in Fig. 4 b. Similarly, as many as 16 enzymes and 7 transporter proteins were observed to be increased in DRYaqlip vs. CTRL, as shown in Fig. 4c. Besides, many of the differentially expressed proteins in all the DES subgroups were annotated as other, many of them of unknown characteristics, namely, PRR4 and zymogen granule protein 16 homolog B (ZG16B). The summary of the PPI networks of the differentially expressed proteins according to their GOCC terms and molecule 
(a) DRYlip vs. CTRL

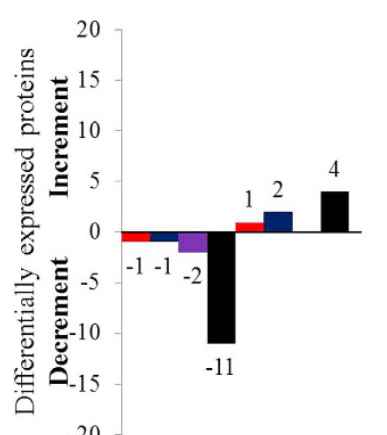

$-20$ (b) DRYaq vs. CTRL

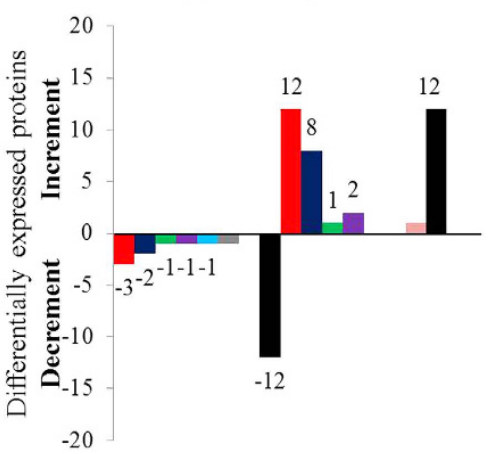

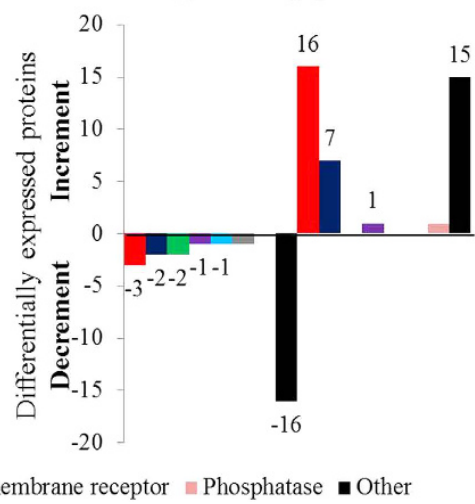

Figure 4. The bar charts shows the over-represented molecular type terms associated with the differentially expressed proteins analysed employing the Ingenuity Pathways Analysis software in (a) DRYlip vs. CTRL, (b) DRYaq vs. CTRL and (c) DRYaqlip vs. CTRL.

types characteristics are as shown in Fig. 5 (the complete lists of PPI networks for each comparison are reported in Supplementary data 1D). Figure 5a demonstrated that there are only 20 direct PPI networks between cytoplasm and extracellular space proteins in DRYlip vs. CTRL. On the contrary, as many as 74 and 169 PPI were observed in DRYaq vs. CTRL and DRYaqlip vs. CTRL, as shown in Fig. 5b,c, respectively. In DRYaq vs. CTRL, the proteins with the highest number of direct PPI networks were transthyretin (TTR) (6), S100A8 (3), cathepsin B (CTSB) (3), gelsolin (GSN) (3), polymeric immunoglobulin receptor (PIGR) (3) and 14-3-3 protein sigma (SFN) (3). In DRYaqlip vs. CTRL, the proteins with the highest number of direct PPI were 14-3-3 protein zeta/delta (YWHAZ) (23), protein S100-A9 (S100A9) (9), ALB (8), annexin A1 (ANXA1) (7), LYZ (6) and PIGR (6). Generally, the most significantly altered PPI networks and their related top diseases and functions for the DRYlip vs. CTRL, DRYaq vs. CTRL and DRYaqlip vs. CTRL were "dermatological diseases and conditions, developmental disorders", "cellular movement, immune cell trafficking, metabolic disease" and "antimicrobial response, inflammatory response, humoral immune response", respectively, as tabulated in Table 1.

Verification of the major differentially expressed tear proteins from DES patients. Finally, a rapid and robust approach via in-solution digestion of the pooled tear samples and accurate inclusion mass screening (AIMS) analysis was utilized to verify the major differentially expressed tear proteins in DES subgroups compared to the CTRL. Table 2 shows the detailed AIMS analysis of the signature peptides based on representative precursor ions for specific proteins (complete data in Supplementary data 1E). This analysis ascertained the differentially expressed profiles of 13 proteins in the DES subgroups compared to CTRL. Among the 13 differentially expressed proteins identified, PRR4, ZG16B and proline-rich protein 1 (PROL1) were found to be significantly decreased in both DRYaq and DRYaqlip subgroups but only slightly decreased in abundance in the DRYlip subgroup. SCGB2A1 and deleted in malignant brain tumors 1 protein (DMBT1) were found to be significantly decreased in both DRYaq and DRYaqlip subgroups. Extracellular glycoprotein lacritin (LACRT) was found significantly decreased only in DRYaqlip subgroup. On the contrary, S100A8, S100A9 were found to be significantly increased in both DRYaq and DRYaqlip subgroups but only slightly increased in abundance in the DRYlip subgroup. Alpha-enolase (ENO1), serotransferrin (TF), phosphatidylethanolamine-binding protein 1 (PEBP1) and alpha-1-acid glycoprotein 1 (ORM1) were found to be significantly increased in both DRYaq and DRYaqlip subgroups. Aldehyde dehydrogenase, dimeric NADP-preferring (ALDH3A1) was found significantly increased only in DRYaqlip subgroup. Two signature peptides for PRR4 were utilized in the AIMS analysis because these two peptides are essential for exact identification and quantification of this protein ${ }^{35}$.

\section{Discussion}

This study had unravelled as many as 79 differentially expressed tear proteins in the DES subgroups compared to CTRL based on the LFQ analysis. Similar expression profiles of the 37 of proteins from this list were already demonstrated in other studies associated with dry eye with/without systemic diseases, as tabulated in Supplementary data 1F, thereby, corroborating with the present results. This study also identified 42 novel proteins associated with DES, and this supports the relevance of the quantitative tear proteomics to identify novel differentially expressed proteins for specific DES.The functional classification and protein interaction network analysis of the significantly altered proteins suggest that the development of DES is a complicated process involving proteins of multiple biological functions. In this study, although 22 tear proteins were differentially expressed in DRYlip vs. CTRL, they were only differentially expressed in a lesser degree and most of them are involved in the dermatological diseases and conditions. On the contrary, the differentially expressed proteins in DRYaq vs. CTRL and DRYaqlip vs. CTRL demonstrated the involvement of mostly extracellular space and cytoplasm proteins, which consisted largely of enzymes and transporter protein types. Basically, the PPI networks analysis demonstrated the involvement of various biological processes and diseases, especially the cellular movement, immune cell trafficking, antimicrobial response, inflammatory response, humoral immune response, metabolic disease and neurological disease. Based on the outcomes of this study, it could be postulated that the pathological 
(a)

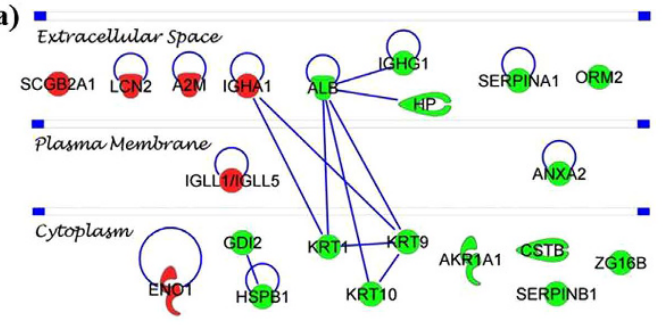

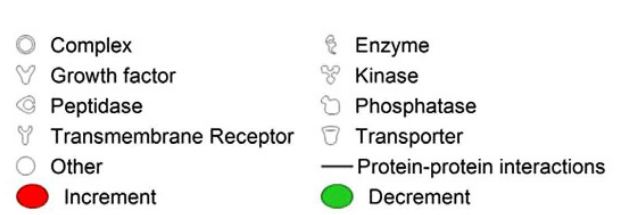

(c)

(b)

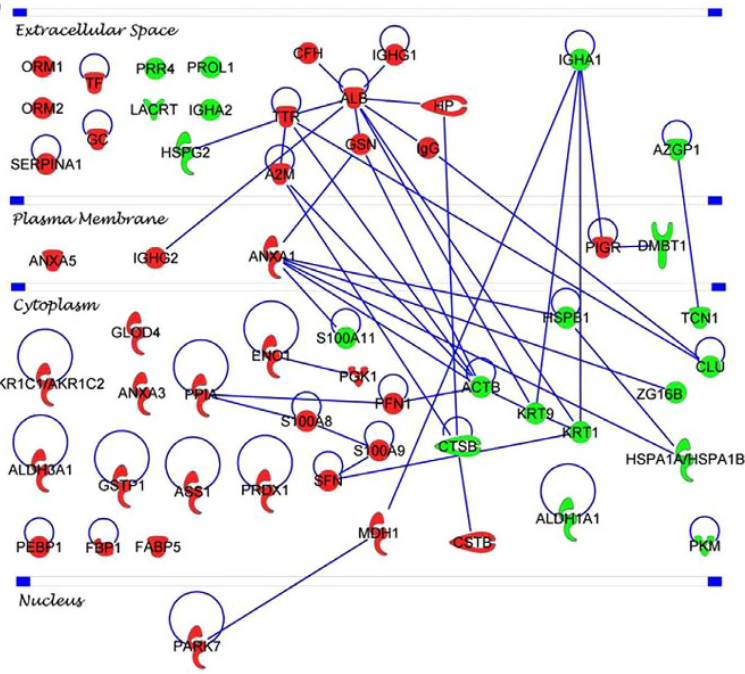

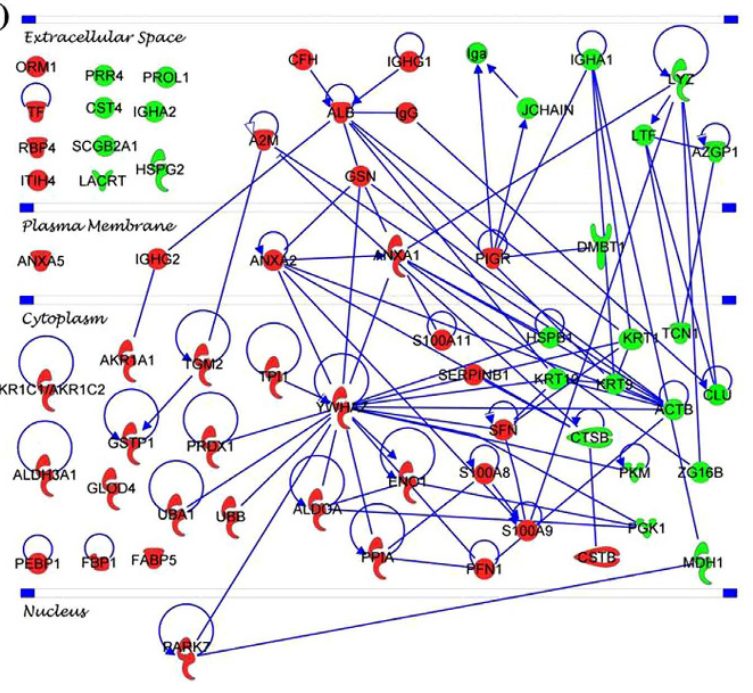

Figure 5. Networks of PPI of the significantly differentially expressed tear proteins analysed employing the Ingenuity Pathways Analysis software in (a) DRYlip vs. CTRL, (b) DRYaq vs. CTRL and (c) DRYaqlip vs. CTRL.

mechanisms underlying DRYaq and DRYaqlip are driven by tear film instability caused largely by the decrement of specific extracellular space and cytoplasm proteins, which mainly induces the activation of inflammatory, immune defence, cell death and apoptosis mechanisms. These host-defence mechanisms act in concert to maintain homeostasis of the tear film following the disease insult ${ }^{1}$. These diverse functions are bio-energetically expensive and require precise control of cellular metabolic pathways ${ }^{39}$. Collectively, these results reveal for the first time that high numbers of metabolic enzymes are expressed, preferentially to fuel the cell fate decisions and effector functions of the aforementioned metabolic processes.

In this study, for the first time, the $\mathrm{iBAQ}$ analysis demonstrated that the top 20 abundant proteins are accountable for approximately $90 \%$ of the total abundance of the tear proteome. This analysis also revealed that most of the significantly differentially expressed proteins were classified as low abundant proteins. For that reason, only 13 major proteins were successfully verified employing the AIMS strategy in DES subgroups compared to CTRL, which composed of PRR4, ZG16B, SCGB2A1, DMBT1, PROL1, LACRT, ALDH3A1, ENO1, TF, S100A8, S100A9, PEBP1 and ORM1. Drastic decrement in abundance of truncated PRR4 in the DRYaq subgroup has been demonstrated previously $y^{6,21,24,26}$. In addition, Boehm et al. and Nichols et al. have demonstrated similar decrement profile of PRR4 in the DRYaqlip subgroup and in contact lens users associated with DES (DRY_CL), respectively ${ }^{20,21}$. Decreased PRR4 expression level was also widely documented in recent studies of DES associated with systemic diseases, namely SS, Stevens-Johnson syndrome (SJS) and rheumatoid arthritis (RA) ${ }^{6,725}$. It is noteworthy that similar decrement patterns of PRR4 were documented in the tears of patients with other systemic diseases such as in diabetic proliferative retinopathy, thyroid-associated orbitopathy and multiple sclerosis ${ }^{40-42}$. Interestingly, although the decreased expression profiles of PRR4 is well documented to be associated with DES and the attribution to the functional relevance of the lacrimal gland, the exact biological functions of this protein in tears remains to be characterized. In addition, our recent study had characterized PRR4 as a complex protein with multiple isoforms and post-translational modifications, which provides an fundamental information for determination of the functional consequences in the disease state ${ }^{35}$. On the other hand, the decrement of ZG16B was only demonstrated by Srinivasan et al. in tears of MSDE patients ${ }^{24}$. In addition, Salvisberg et al. reported decrement of ZG16B in tears of patients with multiple sclerosis ${ }^{42}$. However, both studies did not verify the ZG16B expression profiles employing orthogonal methods, mainly owing to the unavailability of specific functional antibodies. The precise biological function of ZG16B is also largely unknown in tears. Up till now, ZG16B is known as a secretory lectin protein that is proposed to play a regulatory role in intestinal goblet cells and in the pancreatic acinar cells to stimulate "flushing out" of the granule content during exocytosis ${ }^{43,44}$. Furthermore, this secreted protein was also assumed to play an important role in the maintenance of the inflammatory state in cancer tissue ${ }^{45-49}$. It is important to highlight here that similar to the findings of this study, both ZG16B and PRR4 were found to be distinctly decreased in the tear samples of patients with multiple sclerosis, a progressive neurological disability processes ${ }^{42}$. 


\begin{tabular}{|c|c|c|c|c|c|}
\hline Groups & Increment & Decrement & Score & Focus molecules & Top diseases and functions \\
\hline \multirow[t]{2}{*}{ DRYlip vs. CTRL } & IGHA1, SCGB1D1, SCGB2A1 & $\begin{array}{l}\text { ALB, CSTB, HP, IGHG1, } \\
\text { KRT1, KRT9, KRT10, ORM2, } \\
\text { SERPINA1 }\end{array}$ & 30 & 12 & $\begin{array}{l}\text { Dermatological diseases and } \\
\text { conditions, developmental disorders }\end{array}$ \\
\hline & A2M, ENO1, IGLL1/IGLL5, LCN2 & $\begin{array}{l}\text { AKR1A1, ANXA2, GDI2, HP, } \\
\text { HSPB1, SERPINB1, ZG16B }\end{array}$ & 27 & 11 & $\begin{array}{l}\text { Digestive system development and } \\
\text { function }\end{array}$ \\
\hline \multirow{3}{*}{ DRYaq vs. CTRL } & $\begin{array}{l}\text { ALB, ANXA1, ANXA5, CFH, } \\
\text { GC, GSN, HP, IGHG1, IGHG2, } \\
\text { MDH1, ORM1, ORM2, PFN1, } \\
\text { PPIA, S00A8, S100A9, SERPINA1, } \\
\text { TF, TTR }\end{array}$ & $\begin{array}{l}\text { ACTB, CLU, HSPA1A/HSPA1B, } \\
\text { HSPG2, IGHA1, KRT1, KRT9, } \\
\text { S100A9, ZG16B }\end{array}$ & 71 & 28 & $\begin{array}{l}\text { Cellular movement, immune cell } \\
\text { trafficking, metabolic disease }\end{array}$ \\
\hline & $\begin{array}{l}\text { A2M, ASS1, CSTB, FABP5, FBP1, } \\
\text { GLOD4, PIGR }\end{array}$ & $\begin{array}{l}\text { CTSB, DMBT1, HSPB1, IGHA2, } \\
\text { LACRT, PKM, PRR4, SCGB1D1 }\end{array}$ & 31 & 15 & $\begin{array}{l}\text { Hereditary disorder, neurological } \\
\text { disease, organismal injury and } \\
\text { abnormalities }\end{array}$ \\
\hline & $\begin{array}{l}\text { AKR1C1/AKR1C2, ALDH3A1, } \\
\text { ANXA3, ENO1, GSTP1, PARK7, } \\
\text { PEBP1, PGK1, PRDX1, SFN }\end{array}$ & ALDH1A1, AZGP1, TCN1 & 26 & 13 & $\begin{array}{l}\text { Small molecule biochemistry, } \\
\text { cancer, endocrine system disorders }\end{array}$ \\
\hline \multirow{3}{*}{ DRYaqlip vs. CTRL } & $\begin{array}{l}\text { ALB, ANXA1, CFH, IGHG1, } \\
\text { IGHG2, ITIH4, ORM1, PARK7, } \\
\text { PIGR, RBP4, S100A8, S100A), } \\
\text { S100A11, TF, TPI1, YWHAZ }\end{array}$ & $\begin{array}{l}\text { AZGP1, CLU, DMBT1, HSPG2, } \\
\text { IGHA1, KRT1, KRT9, KRT10, } \\
\text { LTF, LYZ, MDH1, TCN1 }\end{array}$ & 73 & 29 & $\begin{array}{l}\text { Antimicrobial response, } \\
\text { inflammatory response, humoral } \\
\text { immune response }\end{array}$ \\
\hline & $\begin{array}{l}\text { A2M, ALDOA, ANXA2, ANXA5, } \\
\text { CSTB, ENO1, GSN, GSTP1, PFN1, } \\
\text { PPIA, SERPINB1, SFN, TGM2 }\end{array}$ & ACTB, CTSB, HSPB1, PGK1 & 36 & 17 & $\begin{array}{l}\text { Cellular movement, inflammatory } \\
\text { response, neurological disease }\end{array}$ \\
\hline & $\begin{array}{l}\text { AKR1A1, FABP5, GLOD4, PEBP1, } \\
\text { PRDX1 }\end{array}$ & $\begin{array}{l}\text { CST4, LACRT, LYZ, PKM, PRR4, } \\
\text { SCGB1D1, SCGB2A1, ZG16B }\end{array}$ & 25 & 13 & $\begin{array}{l}\text { Carbohydrate metabolism, } \\
\text { small molecule biochemistry, } \\
\text { cardiovascular disease }\end{array}$ \\
\hline
\end{tabular}

Table 1. The list of the significantly altered PPI networks and their related top diseases and functions analysed employing the Ingenuity Pathways Analysis software in DES subgroups compared to CTRL.

On the contrary, in our recent study, both PRR4 and ZG16B were found to be significantly increased in abundance in reflex tears, a stimulus by neurological processes ${ }^{50}$. Taken together, the decrement of PRR4 and ZG16B in tears of DES patients could be hypothesized to be associated with the impairment of the neurological processes in the lacrimal gland. Next, similar to the findings of the present study, Srinivasan et al. also documented decrement of PROL1 in the MSDE ${ }^{24}$. The exact function of PROL1 is also still largely unknown. Recently, Dufour et al. reported that the immunoreactive opiorphin (QRFSR-peptide), a mature secretory peptide product of the PROL1 gene, is secreted primarily by lacrimal gland tears at the highest physiological rates $(\sim 200 \mathrm{ng} / \mathrm{ml})$ in healthy volunteers ${ }^{51}$. It has been suggested that the potential role of opiorphin is in modulating lacrimal fluid homeostasis by increasing enkephalin bioavailability in case of certain causes of epiphora. Opiorphin has also been proposed to play paracrine and/or autocrine roles in the lacrimal system and at the ocular surface. Similar decrement in abundance of SCGB2A1 in DRYaq group was also confirmed by Soria et al. and Srinivasan et al. ${ }^{22,24}$. Although the exact physiological function of SCGB2A1 is still not known, it is hypothesized to play a crucial role as an anti-inflammatory agent owing to its association as a member of the uteroglobin family ${ }^{52}$. Next, DMBT1 was found for the first time to be significantly decreased in abundance in DRYaq and DRYaqlip subgroups in this study. It is well documented that this protein plays critical roles in the epithelial differentiation, cellular immune defence and mucosal defence system ${ }^{53-55}$. Decrement abundance of LACRT was also documented in various studies associated with DES, namely in the DRYaq, SS, SJS, RA, MDE, MSDE and DRY_CL $6,20,24,25$. Recent studies demonstrated that this secretory glycoprotein might play an imperative function in secretion and renewal of ocular surface and lacrimal epithelia ${ }^{56,57}$. In this study, protein S100A8 and S100A9 were found to be increased in abundance in all the DES subgroups, and they discriminated best the DRYaq and DRYaqlip from the CTRL. Decrement in abundance of S100A8 and S100A9 were widely documented in various studies associated with DRYaq, DRYaqlip and $\mathrm{SS}^{21-23,25,26}$. These proteins are identified to play a part in inflammatory processes and Zhou et al. demonstrated that higher expressions of these proteins were related with increased signs of dryness ${ }^{23}$. Zhou et al. also demonstrated that the increased abundance of ENO1 in the tears is correlated to the DES ${ }^{23}$. ENO1 is an important glycolytic enzyme, hitherto several studies demonstrated their potential roles in several disease progressions, namely, in cancer and autoimmune disorder ${ }^{58-61}$. The similar increment of ORM1 protein was also documented in DRYaqlip and SS groups ${ }^{23,25}$. ORM1, is a heavily glycosylated protein and categorized as a member of the immunocalin family that modulates inflammatory and immune responses ${ }^{62}$. Meanwhile, the similar increment of TF was only documented by Li et al. in DRY_SS group ${ }^{25}$. TF is an iron binding transport protein and this characteristic resulted in anti-microbial properties ${ }^{63,64}$. PEBP1 was found for the first time significantly increased in abundance in DRYaq and DRYaqlip groups in this study. PEBP1 is an inhibitory modulator of Raf kinase protein and G-protein coupled receptor (GPCR) signalling cascade, as well as an activator of nuclear factor $\kappa \mathrm{B}(\mathrm{NF}-\kappa \mathrm{B})^{65,66}$. Therefore, PEBP1 basically represents a novel effector of signal transduction pathways that modulate apoptosis, motility, therapeutic resistance, genomic integrity and cellular growth ${ }^{65}$. Besides, the disruption of PEBP1 has been reported to be related with a diverse range of diseases, namely, pancreatitis, cancer and Alzheimer's disease, making it a potential target for disease therapy ${ }^{65}$. Increment in abundance of ALDH3A1 was also documented in several studies of DRY_SS, MDE but has never been verified ${ }^{24,25}$. ALDH3A1 is a corneal crystallin protein, highly expressed in epithelial cells and stromal keratocytes ${ }^{67}$. ALDH3A1 plays an active metabolism 


\begin{tabular}{|c|c|c|c|c|c|c|c|c|c|c|c|}
\hline \multirow[b]{3}{*}{ Gene names } & \multirow[b]{3}{*}{ Peptides [AA-sequences] } & \multirow{3}{*}{$\begin{array}{l}\mathbf{m} / \mathbf{z} \\
{[\mathrm{Da}]}\end{array}$} & \multirow{3}{*}{$\begin{array}{c}\text { Charge } \\
{[+]}\end{array}$} & \multirow[b]{3}{*}{$\mathrm{MZ}+[\mathrm{Da}]$} & \multirow[b]{3}{*}{ Score } & \multicolumn{6}{|c|}{ Two samples t-test; (*Significant $P<0.05$ ) } \\
\hline & & & & & & \multicolumn{2}{|c|}{ DRYlip vs. CTRL } & \multicolumn{2}{|c|}{ DRYaq vs. CTRL } & \multicolumn{2}{|c|}{ DRYaqlip vs. CTRL } \\
\hline & & & & & & Significant & p value & Significant & p value & Significant & p value \\
\hline \multirow{2}{*}{ PRR4 } & FPSVSLQEASSFFQR & 865.43 & 2 & 1728.847 & 344 & \multirow{2}{*}{ decrement } & \multirow{2}{*}{0.0004} & \multirow{2}{*}{ decrement } & \multirow{2}{*}{0.0000} & \multirow{2}{*}{ decrement } & \multirow{2}{*}{0.0000} \\
\hline & FPSVSLQEASSFFR & 801.40 & 2 & 1600.789 & 340 & & & & & & \\
\hline ZG16B & YFSTTEDYDHEITGLR & 973.94 & 2 & 1945.869 & 127 & decrement & 0.0016 & decrement & 0.0000 & decrement & 0.0000 \\
\hline SCGB2A1 & TINSDISIPEYK & 690.35 & 2 & 1378.698 & 197 & non-significant & 0.0588 & decrement & 0.0008 & decrement & 0.0000 \\
\hline DMBT1 & FGQGSGPIVLDDVR & 730.38 & 2 & 1458.747 & 187 & non-significant & 0.1682 & decrement & 0.0228 & decrement & 0.0000 \\
\hline PROL1 & FSQAVILSQLFPLESIR & 974.55 & 2 & 1947.083 & 200 & decrement & 0.0081 & decrement & 0.0001 & decrement & 0.0000 \\
\hline LACRT & SILLTEQALAK & 593.85 & 2 & 1185.697 & 255 & non-significant & 0.3401 & non-significant & 0.0521 & decrement & 0.0436 \\
\hline ALDH3A1 & SLEEAIQFINQR & 724.38 & 2 & 1446.747 & 135 & non-significant & 0.1853 & non-significant & 0.3497 & increment & 0.0083 \\
\hline ENO1 & GNPTVEVDLFTSK & 703.86 & 2 & 1405.709 & 155 & non-significant & 0.2672 & increment & 0.0256 & increment & 0.0037 \\
\hline TF & HSTIFENLANK & 637.33 & 2 & 1272.646 & 84 & non-significant & 0.3587 & increment & 0.0000 & increment & 0.0000 \\
\hline S100A8 & ALNSIIDVYHK & 636.85 & 2 & 1271.687 & 142 & increment & 0.0229 & increment & 0.0004 & increment & 0.0000 \\
\hline PEBP1 & GNDISSGTVLSDYVGSGPPK & 975.48 & 2 & 1948.938 & 98 & non-significant & 0.7171 & increment & 0.0007 & increment & 0.0000 \\
\hline ORM1 & YVGGQEHFAHLLILR & 876.98 & 2 & 1751.947 & 127 & non-significant & 0.4548 & increment & 0.0004 & increment & 0.0000 \\
\hline S100A9 & NIETIINTFHQYSVK & 903.97 & 2 & 1805.931 & 342 & increment & 0.0001 & increment & 0.0000 & increment & 0.0000 \\
\hline
\end{tabular}

Table 2. Summary of the significantly differentially expressed proteins in DES subgroups compared to CTRL employing the targeted proteomics strategy.

of toxic aldehydes in the cornea ${ }^{68}$. An essential role for ALDH3A1 in the cornea is suggested by observation that diseased corneas, especially cataract development, are associated with decreased ALDH3A1 catalytic activity 69,70 .

The rest of the 66 differentially expressed proteins were not successfully verified, largely due to the low abundance of these proteins in tears and limitations of the AIMS strategy. It is well-recognized that in the sequence of new biomarker identification, the validity of potential candidate biomarker panels must be tested thoroughly in individual samples. However, biomarker validation is a very costly and time-consuming task, primarily due to the complexity of multiplexed assays, as well as technological challenges in identifying and quantifying low abundant proteins $^{71,72}$. Hence, in the current study, the AIMS analysis employed was limited to the pooled tear samples to screen for and verify major differentially expressed proteins in DES samples. However, since this is the first study that extensively identified and verified the cluster of differentially expressed proteins in tears, which could be potential biomarker candidate(s) for the different subgroups of DES. The important findings emerging from this this study with the use of pooled samples are envisioned to provide fundamental information and will be a highly useful platform and reference point for future studies utilizing individual samples to dissect 'personalized' expression of these proteins. This will be especially useful for analysis of individual tear samples of larger cohorts of DES patients in the clinical settings to demonstrate the discriminative power (e.g. sensitivity and specificity).

In conclusion, this study had unravelled the intricate regulation profiles of specific cluster of proteins responsible for the maintenance of tear film stability in the different DES subgroups. Besides, we have unravelled numerous proteins that participate in metabolic processes, which might provide new insights for modulating various biological processes, especially the inflammatory and immune defence disorders of the ocular surface. The outcomes of the identification of these proteins, when extrapolated to clinical application, can provide invaluable hints on development of specific diagnostic tool for clinical tests and are of great importance for the prognostic usage for improved clinical management of the disease in the future.

\section{Materials and Methods}

Tear sampling. The study was performed in strict adherence to the guidelines of the 1964 Declaration of Helsinki and all experimental protocols were approved by the "Landesärztekammer Rheinland-Pfalz" ethics committee. All participants were informed of the possible risks, the goal of the study and privacy policy, and an informed consent was signed according to the recommendations of the Declaration of Helsinki for investigation with human subjects. In this study, tear proteins of 80 patients were included and assigned into DRYlip, DRYaq, DRYaqlip and CTRL. Each group comprises 20 subjects equally divided to male (M) and female (F), age between 21 to 79 years old. The classification of patients was carried out according to the guidelines of the Tear Film and Ocular Surface Society (TFOS, Ocular Surface, 2007) at the Department of Ophthalmology at the University Medical Center of the Johannes Gutenberg University, Mainz, following specific inclusion and exclusion criteria of basic secretory test (BST), tear breakup time (TBUT), clinical parameters after Bron and Foulks Score and Lid-parallel Conjunctival Folds (LIPCOF) and extensively asking for the anamnesis and symptoms using Ocular Surface Disease Index (OSDI) questionnaire. Table 3 summarizes the clinical evaluation parameters for the classification DES subgroups and CTRL patients (complete description in Supplementary data 1G). From each individual, tear samples have been taken by a BST, a clinical standard test, using Schirmer's strip. Schirmer's strips have been placed into the lower eye lid for a few minutes, after having prepared the eye with mild anesthetic eye drops (Novesine). The eye drops were used in this study to assess the basal tear secretion from the patients as critically as possible. The samples were immediately stored at $-80^{\circ} \mathrm{C}$ for subsequent analysis. Tear proteins were extracted from the Schirmer's strips by soaking each strip in $500 \mu \mathrm{l}$ phosphate-buffered saline (PBS) for 3 hours at $4{ }^{\circ} \mathrm{C}$ to elute the tear proteins. Subsequently, the total protein concentrations in the collected tear samples were 


\begin{tabular}{|c|c|c|c|c|c|c|c|}
\hline \multirow[b]{2}{*}{ DES group } & \multicolumn{3}{|c|}{ Clinical parameters } & \multirow[b]{2}{*}{ No. Patients } & \multirow[b]{2}{*}{ Gender } & \multirow[b]{2}{*}{ Age } & \multirow[b]{2}{*}{ Protein Conc. $(\mu \mathrm{g} / \mu \mathrm{l})$} \\
\hline & BST & TBUT & Bron and Foulks Score & & & & \\
\hline \multirow{2}{*}{ CTRL } & \multirow{2}{*}{$>10 \mathrm{~mm} / 5 \mathrm{~min}$} & \multirow{2}{*}{$>10 \mathrm{~s}$} & \multirow{2}{*}{$<18$} & 10 & Male & $51.4 \pm 14.78$ & $0.28 \pm 0.14$ \\
\hline & & & & 10 & Female & $43.8 \pm 15.44$ & $0.32 \pm 0.10$ \\
\hline \multirow{2}{*}{ DRYlip } & \multirow{2}{*}{$>10 \mathrm{~mm} / 5 \mathrm{~min}$} & \multirow{2}{*}{$<10 \mathrm{~s}$} & \multirow{2}{*}{$>18$} & 10 & Male & $52.9 \pm 20.45$ & $0.23 \pm 0.09$ \\
\hline & & & & 10 & Female & $51.8 \pm 18.66$ & $0.28 \pm 0.06$ \\
\hline \multirow{2}{*}{ DRYaq } & \multirow{2}{*}{$<10 \mathrm{~mm} / 5 \mathrm{~min}$} & \multirow{2}{*}{$>10 \mathrm{~s}$} & \multirow{2}{*}{$<18$} & 10 & Male & $47.6 \pm 15.32$ & $0.18 \pm 0.07$ \\
\hline & & & & 10 & Female & $49.6 \pm 14.74$ & $0.17 \pm 0.07$ \\
\hline \multirow{2}{*}{ DRYaqlip } & \multirow{2}{*}{$<10 \mathrm{~mm} / 5 \mathrm{~min}$} & \multirow{2}{*}{$<10 \mathrm{~s}$} & \multirow{2}{*}{$>18$} & 10 & Male & $57.73 \pm 19.38$ & $0.19 \pm 0.09$ \\
\hline & & & & 10 & Female & $58.78 \pm 17.42$ & $0.15 \pm 0.06$ \\
\hline
\end{tabular}

Table 3. Summary of the clinical parameters for the classification of DES subgroups and CTRL patients.

determined using BCA Protein Assay Kit (Pierce, Rockford, IL) prior to further analysis. Supplementary data $1 \mathrm{H}$ summarizes the general description of the study samples and the total protein concentration yielded from DES subgroups and CTRL patients.

Discovery study: LFO analysis via 1DE \& LC-ESI-MS/MS strategy. Label-free quantification of peptides via 1DE \& LC-ESI-MS/MS strategy was employed to identify the change in protein abundance in the discovery data and to generate a list of the differentially expressed proteins from specific DES subgroups for subsequent verification. The tear samples for each assigned group $(\mathrm{N}=20)$ were pooled equally $(2.5 \mu \mathrm{g}$ per individual) to a total of $50 \mu \mathrm{g}$ with three replicates. The reason for equal amount of protein collection and pooling from each patient within a group was to normalize the difference between subjects and to reduce individual variation. The pooled tear samples of each DES subgroups were subjected to $1 \mathrm{DE}(50 \mu \mathrm{g} / \mathrm{well}$ and sliced into 10 bands), trypsin digested and the extracted peptides were purified utilizing methods previously described ${ }^{35,50}$. The LC-ESI-LTQ-Orbitrap MS system employed and the mass spectrometric settings utilized for this study has been described in detail elsewhere ${ }^{35,50}$. The acquired continuum MS spectra were analysed by MaxQuant computational proteomics platform version 1.4.1.2 and its built-in Andromeda search engine for peptide and protein identification, with LFQ and iBAQ algorithm enabled ${ }^{38,73-75}$. The tandem MS spectra were searched against Uniprot Human database (date, 19/March/2014) using settings as described in detail elsewhere ${ }^{50}$. The false discovery rate (FDR) for protein identification was set to 0.01 with $\geq 6$ amino acid residues and "unique plus razor peptides" were selected to be included for LFQ and iBAQ analysis ${ }^{73}$. The output of the generated "proteingroups. txt" data from the MaxQuant analysis was utilized for Pearson correlation, clustering and statistical analysis using Perseus software. Unsupervised hierarchical clustering of the LFQ values was performed based on Euclidean distances on the Z-scored between mean values. For statistical analysis, two-samples t-test-based statistics with $\mathrm{P}<0.01$ was applied on $\log 2$ transformed LFQ values and the minimum number of values "in at least one group" is 3 to assert proteins regulation as significant for the specific groups ${ }^{38,50}$. The iBAQ values, which were calculated by dividing the summed peptide intensities for a given protein with the number of theoretically observable tryptic peptides, act as a degree of protein abundance were converted to percentage and employed to compare between proteins in each group.

Verification Study: Targeted MS via AIMS strategy. The identified candidate biomarkers from the discovery stage were verified employing a targeted form of MS strategy called AIMS ${ }^{71,76}$. The main aim of employing the AIMS strategy for verification in the present study is to determine the major differentially expressed proteins to discriminate the specific DES subgroups. The tear samples for each assigned group $(\mathrm{N}=20)$ were pooled equally $(0.5 \mu \mathrm{g}$ per individual) to a total of $10 \mu \mathrm{g}$ with quadruplicate and subjected to in-solution digestion. The pooled tear samples were digested with sequence grade-modified trypsin [protease: protein ratio of 1:20 (w/w)] for 16 hours at $37^{\circ} \mathrm{C}$ with $50 \mu \mathrm{l}$ of $50 \mathrm{mM} \mathrm{NH}_{4} \mathrm{HCO}_{3}$ in $10 \%$ acetonitrile. The digested samples were purified on ZipTip C18 columns and the eluates were concentrated to dryness in the SpeedVac concentrator and dissolved with $10 \mu \mathrm{l}$ of $0.1 \%$ TFA solution prior to targeted MS analysis ${ }^{35,50}$. For targeted MS analysis, the LC-ESI-MS/MS system was operated as described in detail previously with several parameters adjusted to target only a specific set of peptides ${ }^{35,50}$. The selection of the experimentally identified peptides list that annotated to specific protein was carried out manually from the "msms.txt" file resulting from MaxQuant analysis governed by these criterions, contains no missed cleavages, fully tryptic, no modifications and peptide only assume charge +2 . The created peptide inclusion list comprising $\mathrm{m} / \mathrm{z}$ and charge was assigned to the instrument acquisition software before data acquisition. The in-solution digested tear samples consisted of very complex mixtures especially the high abundant proteins. Hence, to yield optimum results, two LC gradients of $60 \mathrm{~min}$ (as described elsewhere ${ }^{35}$ ) and $120 \mathrm{~min}$ were utilized. The gradient for $120 \mathrm{~min}$ per sample is as follows: $0-5 \mathrm{~min}$ : $10 \% \mathrm{~B}, 5-95 \mathrm{~min}: 10-50 \% \mathrm{~B}$, 95-105 min: $50-90 \%$ B, 105-110 min: $90 \%$ B, 110-115 min: 90-10\% B, 115-120 min: 10\% B. The adjusted parameters for inclusion list-dependent acquisition were as follows: the dynamic exclusion segment was disabled, the use of global parent list was enabled and the $m / z$ tolerance around targeted precursors was $\pm 10 \mathrm{ppm}$. The acquired MS spectra were analysed by MaxQuant for peptide identification by searched against Uniprot Human database (date, 19/March/2014) using settings with peptide mass tolerance of $\pm 10 \mathrm{ppm}$, fragment mass tolerance of \pm 0.5 , peptide charge state of +2 and FDR for peptide identification was set to 0.01 . The output data of the generated 
'peptides.txt' file from MaxQuant were used to calculate the sum absolute intensity of the signature peptides for each proteins, and were transferred to Statistica (v8, StatSoft, Tulsa, OK) for t-test analysis (independent, by groups, $\mathrm{P}<0.05)$.

Functional annotation and pathways analysis. Proteins determined to be differentially expressed as described based on the data in our LFQ experiments were tabulated in Excel and their gene names were used for functional annotation and pathways analysis. First, DAVID tool (version 6.7) (http://david.abcc.ncifcrf.gov/ home.jsp) was used for interpreting the GOBP terms of the differentially-expressed proteins $\mathrm{s}^{77,78}$. The protein list was uploaded into DAVID and searched for enrichment for GOBP term and the results were filtered based on threshold count $\geq 2$ and $P$ values $<0.05$. Ingenuity Pathways Analysis software (IPA, Ingenuity QIAGEN Redwood City, CA) (www.qiagen.com/ingenuity) was used for interpreting the GOCC terms, molecule types and PPI networks as well as top diseases and functions associated with the differentially-expressed proteins. Top canonical pathways involving the differentially expressed proteins were presented, along with a $p$-value calculated using Fisher's exact test. The molecular interactions networks between proteins associated with top diseases and functions were reported. Proteins are displayed with their corresponding gene names and represented as nodes, whereas protein-protein interactions based on direct associations (experimentally observed) between two nodes are represented with an edge (line). Nodes are presented using different shapes to represent the functional protein class and node colour indicates decreased (green) or increased (red) abundance.

\section{References}

1. DEWS. The definition and classification of dry eye disease: report of the Definition and Classification Subcommittee of the International Dry Eye WorkShop Ocul Surf 5, 75-92 (2007).

2. Tomosugi, N., Kitagawa, K., Takahashi, N., Sugai, S. \& Ishikawa, I. Diagnostic potential of tear proteomic patterns in Sjögren's syndrome. Journal of proteome research 4, 820-825 (2005).

3. Tsai, P. S. et al. Proteomic analysis of human meibomian gland secretions. Br J Ophthalmol 90, 372-377, doi: 10.1136/ bjo.2005.080846 (2006).

4. Li, K., Liu, X., Chen, Z., Huang, Q. \& Wu, K. Quantification of tear proteins and sPLA2-IIa alteration in patients with allergic conjunctivitis. Molecular vision 16, 2084 (2010).

5. Zhou, L. et al. Elevation of human alpha-defensins and S100 calcium-binding proteins A8 and A9 in tear fluid of patients with pterygium. Invest Ophthalmol Vis Sci 50, 2077-2086, doi: 10.1167/iovs.08-2604 (2009).

6. Aluru, S. V. et al. Lacrimal proline rich 4 (LPRR4) protein in the tear fluid is a potential biomarker of dry eye syndrome. PLoS One 7, e51979, doi: 10.1371/journal.pone.0051979 (2012).

7. Saijyothi, A. V. et al. Two dimensional electrophoretic analysis of human tears: collection method in dry eye syndrome. Electrophoresis 31, 3420-3427, doi: 10.1002/elps.201000271 (2010).

8. Schein, O. D., Munoz, B., Tielsch, J. M., Bandeen-Roche, K. \& West, S. Prevalence of dry eye among the elderly. American journal of ophthalmology 124, 723-728 (1997).

9. Jie, Y., Xu, L., Wu, Y. \& Jonas, J. Prevalence of dry eye among adult Chinese in the Beijing Eye Study. Eye 23, 688-693 (2008).

10. Guo, B., Lu, P., Chen, X., Zhang, W. \& Chen, R. Prevalence of dry eye disease in Mongolians at high altitude in China: the Henan eye study. Ophthalmic epidemiology 17, 234-241 (2010).

11. McCarty, C. A., Bansal, A. K., Livingston, P. M., Stanislavsky, Y. L. \& Taylor, H. R. The epidemiology of dry eye in Melbourne, Australia. Ophthalmology 105, 1114-1119 (1998).

12. Moss, S. E., Klein, R. \& Klein, B. E. Prevalence of and risk factors for dry eye syndrome. Archives of ophthalmology 118, 1264-1268 (2000).

13. Lee, A. et al. Prevalence and risk factors associated with dry eye symptoms: a population based study in Indonesia. British Journal of Ophthalmology 86, 1347-1351 (2002).

14. Viso, E., Rodriguez-Ares, M. T. \& Gude, F. Prevalence of and associated factors for dry eye in a Spanish adult population (the Salnes Eye Study). Ophthalmic epidemiology 16, 15-21 (2009)

15. Lekhanont, K., Rojanaporn, D., Chuck, R. S. \& Vongthongsri, A. Prevalence of dry eye in Bangkok, Thailand. Cornea 25, 1162-1167 (2006).

16. Chia, E. M. et al. Prevalence and associations of dry eye syndrome in an older population: the Blue Mountains Eye Study. Clin Experiment Ophthalmol 31, 229-232 (2003).

17. Hashemi, H. et al. Prevalence of dry eye syndrome in an adult population. Clin Experiment Ophthalmol 42, 242-248, doi: 10.1111/ ceo.12183 (2014).

18. Yu, J., Asche, C. V. \& Fairchild, C. J. The economic burden of dry eye disease in the United States: a decision tree analysis. Cornea 30, 379-387 (2011).

19. Waduthantri, S. et al. Cost of dry eye treatment in an Asian clinic setting. PloS One 7, e37711 (2012).

20. Grus, F. H. et al. SELDI-TOF-MS ProteinChip array profiling of tears from patients with dry eye. Investigative ophthalmology \& visual science 46, 863-876 (2005).

21. Boehm, N. et al. Alterations in the Tear Proteome of Dry Eye Patients-A Matter of the Clinical PhenotypeTear Proteome of Dry Eye Patients. Investigative ophthalmology \& visual science 54, 2385-2392 (2013).

22. Soria, J. et al. Tear proteome and protein network analyses reveal a novel pentamarker panel for tear film characterization in dry eye and meibomian gland dysfunction. J Proteomics 78, 94-112, doi: 10.1016/j.jprot.2012.11.017 (2013).

23. Zhou, L. et al. Identification of tear fluid biomarkers in dry eye syndrome using iTRAQ quantitative proteomics. J Proteome Res $\mathbf{8}$, 4889-4905, doi: 10.1021/pr900686s (2009).

24. Srinivasan, S., Thangavelu, M., Zhang, L., Green, K. B. \& Nichols, K. K. iTRAQ quantitative proteomics in the analysis of tears in dry eye patients. Investigative ophthalmology \& visual science 53, 5052-5059 (2012).

25. Li, B. et al. Tear proteomic analysis of Sjögren syndrome patients with dry eye syndrome by two-dimensional-nano-liquid chromatography coupled with tandem mass spectrometry. Scientific reports 4 (2014).

26. Nichols, J. J. \& Green-Church, K. B. Mass spectrometry-based proteomic analyses in contact lens-related dry eye. Cornea 28, 1109-1117, doi: 10.1097/ICO.0b013e3181a2ad81 (2009).

27. Zhou, L. et al. In-depth analysis of the human tear proteome. J Proteomics 75, 3877-3885, doi: 10.1016/j.jprot.2012.04.053 (2012).

28. Funke, S., Azimi, D., Wolters, D., Grus, F. H. \& Pfeiffer, N. Longitudinal analysis of taurine induced effects on the tear proteome of contact lens wearers and dry eye patients using a RP-RP-Capillary-HPLC-MALDI TOF/TOF MS approach. J Proteomics 75, 3177-3190, doi: 10.1016/j.jprot.2012.03.018 (2012).

29. Lema, I., Brea, D., Rodriguez-Gonzalez, R., Diez-Feijoo, E. \& Sobrino, T. Proteomic analysis of the tear film in patients with keratoconus. Mol Vis 16, 2055-2061 (2010).

30. Ananthi, S. et al. Comparative analysis of the tear protein profile in mycotic keratitis patients. Mol Vis 14, 500-507 (2008). 
31. Green-Church, K. B., Nichols, K. K., Kleinholz, N. M., Zhang, L. \& Nichols, J. J. Investigation of the human tear film proteome using multiple proteomic approaches. Mol Vis 14, 456-470 (2008).

32. de Souza, G. A., Godoy, L. M. \& Mann, M. Identification of 491 proteins in the tear fluid proteome reveals a large number of proteases and protease inhibitors. Genome Biol 7, R72, doi: 10.1186/gb-2006-7-8-R72 (2006).

33. Zhou, L. et al. Characterisation of human tear proteins using high-resolution mass spectrometry. Ann Acad Med Singapore 35, 400-407 (2006).

34. Li, N. et al. Characterization of human tear proteome using multiple proteomic analysis techniques. J Proteome Res 4, 2052-2061, doi: 10.1021/pr0501970 (2005).

35. Perumal, N., Funke, S., Pfeiffer, N. \& Grus, F. H. Characterization of lacrimal proline-rich protein 4 (PRR4) in human tear proteome. Proteomics 14, 1698-1709 (2014).

36. Nahnsen, S., Bielow, C., Reinert, K. \& Kohlbacher, O. Tools for label-free peptide quantification. Molecular \& Cellular Proteomics 12, 549-556 (2013).

37. Bantscheff, M., Lemeer, S., Savitski, M. M. \& Kuster, B. Quantitative mass spectrometry in proteomics: critical review update from 2007 to the present. Anal Bioanal Chem 404, 939-965, doi: 10.1007/s00216-012-6203-4 (2012).

38. Cox, J. et al. MaxLFQ allows accurate proteome-wide label-free quantification by delayed normalization and maximal peptide ratio extraction. Molecular \& Cellular Proteomics, mcp. M113, 031591 (2014).

39. Ganeshan, K. \& Chawla, A. Metabolic regulation of immune responses. Annu Rev Immunol 32, 609-634, doi: 10.1146/annurevimmunol-032713-120236 (2014).

40. Matheis, N., Okrojek, R., Grus, F. H. \& Kahaly, G. J. Proteomics of tear fluid in thyroid-associated orbitopathy. Thyroid 22, 1039-1045, doi: 10.1089/thy.2012.0119 (2012).

41. Csosz, E. et al. Quantitative analysis of proteins in the tear fluid of patients with diabetic retinopathy. J Proteomics 75, 2196-2204, doi: 10.1016/j.jprot.2012.01.019 (2012).

42. Salvisberg, C. et al. Exploring the human tear fluid: discovery of new biomarkers in multiple sclerosis. Proteomics Clin Appl 8, 185-194, doi: 10.1002/prca.201300053 (2014).

43. De Lisle, R. C. \& Hopfer, U. Electrolyte permeabilities of pancreatic zymogen granules: implications for pancreatic secretion. Am J Physiol 250, G489-G496 (1986).

44. Guo, X. W., Merlin, D., Laboisse, C. \& Hopfer, U. Purinergic agonists, but not cAMP, stimulate coupled granule fusion and Clconductance in HT29-Cl.16E. Am J Physiol 273, C804-C809 (1997).

45. Kanagawa, M. et al. Crystal structures of human secretory proteins ZG16p and ZG16b reveal a Jacalin-related beta-prism fold. Biochem Biophys Res Commun 404, 201-205, doi: 10.1016/j.bbrc.2010.11.093 (2011).

46. Neuschwander-Tetri, B. A., Fimmel, C. J., Kladney, R. D., Wells, L. D. \& Talkad, V. Differential expression of the trypsin inhibitor SPINK3 mRNA and the mouse ortholog of secretory granule protein ZG-16p mRNA in the mouse pancreas after repetitive injury. Pancreas 28, e104-e111 (2004).

47. Kim, S. A. et al. Pancreatic adenocarcinoma up-regulated factor (PAUF), a novel up-regulated secretory protein in pancreatic ductal adenocarcinoma. Cancer Sci 100, 828-836, doi: 10.1111/j.1349-7006.2009.01106.x (2009).

48. Lee, Y. et al. PAUF functions in the metastasis of human pancreatic cancer cells and upregulates CXCR4 expression. Oncogene 29, 56-67, doi: 10.1038/onc.2009.298 (2010).

49. Barderas, R. et al. In-depth characterization of the secretome of colorectal cancer metastatic cells identifies key proteins in cell adhesion, migration, and invasion. Mol Cell Proteomics 12, 1602-1620, doi: 10.1074/mcp.M112.022848 (2013).

50. Perumal, N., Funke, S., Wolters, D., Pfeiffer, N. \& Grus, F. H. Characterization of human reflex tear proteome reveals high expression of lacrimal proline-rich protein 4 (PRR4). Proteomics 15, 3370-3381 (2015).

51. Dufour, E. et al. Opiorphin secretion pattern in healthy volunteers: gender difference and organ specificity. Biochem Anal Biochem 2, 2-11 (2013).

52. Benson, M. et al. Gene profiling reveals increased expression of uteroglobin and other anti-inflammatory genes in glucocorticoidtreated nasal polyps. J Allergy Clin Immunol 113, 1137-1143, doi: 10.1016/j.jaci.2004.02.028 (2004).

53. Prakobphol, A. et al. Salivary agglutinin, which binds streptococcus mutansand helicobacter pylori, is the lung scavenger receptor cysteine-rich protein gp-340. Journal of Biological Chemistry 275, 39860-39866 (2000).

54. Mollenhauer, J. et al. Deleted in Malignant Brain Tumors 1 is a versatile mucin-like molecule likely to play a differential role in digestive tract cancer. Cancer research 61, 8880-8886 (2001).

55. Mollenhauer, J., End, C., Renner, M., Lyer, S. \& Poustka, A. DMBT1 as an archetypal link between infection, inflammation, and cancer. Inmunologia 26, 193-209 (2007).

56. McKown, R. L. et al. Lacritin and other new proteins of the lacrimal functional unit. Experimental eye research 88, 848-858 (2009).

57. Karnati, R., Laurie, D. E. \& Laurie, G. W. Lacritin and the tear proteome as natural replacement therapy for dry eye. Experimental eye research 117, 39-52 (2013).

58. Li, J., Qin, J., Zhang, H., Shan, Z. \& Teng, W. OR12-2: Direct Identification of Alpha-Enolase As an Autoantigen in the Pathogenesis of Autoimmune Thyroiditis (2014).

59. Capello, M. et al. Can the moonlighting glycolytic enzyme \{alpha\}-enolase be a therapeutic target in pancreatic cancer. Cancer Research 73, 1889 (2013).

60. Diaz-Ramos, A., Roig-Borrellas, A., Garcia-Melero, A. \& Lopez-Alemany, R. alpha-Enolase, a multifunctional protein: its role on pathophysiological situations. J Biomed Biotechnol 2012, 156795, doi: 10.1155/2012/156795 (2012).

61. Zhou, W. et al. Mass spectrometry analysis of the post-translational modifications of $\alpha$-enolase from pancreatic ductal adenocarcinoma cells. Journal of proteome research 9, 2929-2936 (2010).

62. Hochepied, T., Berger, F. G., Baumann, H. \& Libert, C. $\alpha$ 1-Acid glycoprotein: an acute phase protein with inflammatory and immunomodulating properties. Cytokine \& growth factor reviews 14, 25-34 (2003).

63. Crichton, R. R. \& Charloteaux-Wauters, M. Iron transport and storage. Eur J Biochem 164, 485-506 (1987)

64. Castellano, A. C. et al. Structure-Function Relationship in the Serotransferrin - the Role of the Ph on the Conformational Change and the Metal-Ions Release. Biochemical and Biophysical Research Communications 198, 646-652, doi: 10.1006/bbrc.1994.1094 (1994).

65. Al-Mulla, F., Bitar, M. S., Taqi, Z. \& Yeung, K. C. RKIP: much more than Raf kinase inhibitory protein. Journal of cellular physiology 228, 1688-1702 (2013).

66. Yeung, K. et al. Suppression of Raf-1 kinase activity and MAP kinase signalling by RKIP. nature 401, 173-177 (1999).

67. Pappa, A., Estey, T., Manzer, R., Brown, D. \& Vasiliou, V. Human aldehyde dehydrogenase 3A1 (ALDH3A1): biochemical characterization and immunohistochemical localization in the cornea. Biochem. J 376, 615-623 (2003).

68. Stagos, D., Chen, Y., Cantore, M., Jester, J. V. \& Vasiliou, V. Corneal aldehyde dehydrogenases: multiple functions and novel nuclear localization. Brain research bulletin 81, 211-218 (2010).

69. Gondhowiardjo, T. D. et al. Analysis of corneal aldehyde dehydrogenase patterns in pathologic corneas. Cornea 12, 146-154 (1993).

70. Lassen, N. et al. Multiple and Additive Functions of ALDH3A1 and Aldh1al Cataract Phenotype and Ocular Oxidative Damage in Aldh3a1 (-/-)/Aldh1a1 (-/-) Knock-out mice. Journal of Biological Chemistry 282, 25668-25676 (2007).

71. Jaffe, J. D. et al. Accurate Inclusion Mass Screening A Bridge from Unbiased Discovery to Targeted Assay Development for Biomarker Verification. Molecular \& Cellular Proteomics 7, 1952-1962 (2008). 
72. Maes, E. et al. Proteomics in cancer research: Are we ready for clinical practice? Critical reviews in oncology/hematology 96, 437-448 (2015).

73. Cox, J. \& Mann, M. MaxQuant enables high peptide identification rates, individualized p.p.b.-range mass accuracies and proteomewide protein quantification. Nat Biotechnol 26, 1367-1372, doi: 10.1038/nbt.1511 (2008).

74. Luber, C. A. et al. Quantitative proteomics reveals subset-specific viral recognition in dendritic cells. Immunity 32, 279-289, doi: 10.1016/j.immuni.2010.01.013 (2010).

75. Cox, J. et al. Andromeda: A Peptide Search Engine Integrated into the MaxQuant Environment. Journal of Proteome Research 10, 1794-1805, doi: 10.1021/pr101065j (2011).

76. Savitski, M. M. et al. Targeted data acquisition for improved reproducibility and robustness of proteomic mass spectrometry assays. Journal of the American Society for Mass Spectrometry 21, 1668-1679 (2010).

77. Huang, D. W., Sherman, B. T. \& Lempicki, R. A. Systematic and integrative analysis of large gene lists using DAVID bioinformatics resources. Nature protocols 4, 44-57 (2009).

78. Huang, D. W., Sherman, B. T. \& Lempicki, R. A. Bioinformatics enrichment tools: paths toward the comprehensive functional analysis of large gene lists. Nucleic acids research 37, 1-13 (2009).

\section{Acknowledgements}

The authors thank Dr. Caroline Manicam from the Department of Ophthalmology, University Medical Center of the Johannes Gutenberg University Mainz for her critical appraisal of the manuscript.

\section{Author Contributions}

N.P. designed the study, carried out the experiments, analysed and interpreted the data and wrote the manuscript. S.F. participated in the design of the study and helped draft the manuscript. N.P. contributed essential materials, reagents and analysis tools and helped draft the manuscript. F.H.G. participated in the design of the study, contributed essential materials, reagents, analysis tools and helped draft the manuscript. All authors read and approved the final version of this manuscript.

\section{Additional Information}

Supplementary information accompanies this paper at http://www.nature.com/srep

Competing financial interests: The authors declare no competing financial interests.

How to cite this article: Perumal, N. et al. Proteomics analysis of human tears from aqueous-deficient and evaporative dry eye patients. Sci. Rep. 6, 29629; doi: 10.1038/srep29629 (2016).

(c) (i) This work is licensed under a Creative Commons Attribution 4.0 International License. The images or other third party material in this article are included in the article's Creative Commons license, unless indicated otherwise in the credit line; if the material is not included under the Creative Commons license, users will need to obtain permission from the license holder to reproduce the material. To view a copy of this license, visit http://creativecommons.org/licenses/by/4.0/ 